\title{
A rapid diagnostic multiplex PCR approach for xenomonitoring of human and animal schistosomiasis in a 'One Health' context
}

\author{
Ruben Schols ${ }^{a}$, Hans Carolus ${ }^{a}$, Cyril Hammoud ${ }^{b, c}$, Stephen Mulero ${ }^{d}$, Aspire Mudavanhu $^{e}$ and Tine Huyse ${ }^{a, b, *}$ \\ ${ }^{a}$ Laboratory of Biodiversity and Evolutionary Genomics, University of Leuven, 3000 Leuven, Belgium; ${ }^{b}$ Department of Biology, Royal \\ Museum for Central Africa, Leuvensesteenweg 13, 3080 Tervuren, Belgium; 'Limnology Research Unit, Ghent University, K. L. \\ Ledeganckstraat 35, 9000 Ghent, Belgium; 'Laboratory of Host-Pathogen-Environment Interactions, IHPE UMR 5244, CNRS, University \\ of Perpignan Via Domitia, 66100 Perpignan, France; ${ }^{e}$ Department of Biological Sciences, University of Zimbabwe, Harare, Zimbabwe \\ *Corresponding author: Tel: +32 2769 5372; E-mail: tine.huyse@afrikamuseum.be
}

Received 18 April 2019; revised 21 June 2019; editorial decision 24 June 2019; accepted 00

\begin{abstract}
Studying the epidemiology of schistosomiasis-the most prevalent gastropod-borne human disease and an economic burden for the livestock industry-relies on adequate monitoring tools. Here we describe a molecular assay for detecting human and animal African schistosome species in their planorbid gastropod host (xenomonitoring) using a two-step approach. First, schistosome infections are detected and discriminated from other trematode infections using a multiplex polymerase chain reaction (PCR) that includes a trematode-specific marker (in 18S rDNA), a Schistosoma genus-specific marker (in internal transcribed spacer 2 [ITS2]) and a general gastropod marker (in 18S rDNA) as an internal control. Upon Schistosoma sp. detection, a second multiplex PCR is performed to discriminate among Schistosoma haematobium, Schistosoma mansoni, Schistosoma mattheei and Schistosoma bovis/Schistosoma curassoni/Schistosoma guineensis using markers of differential lengths in the cytochrome c oxidase subunit 1 (COX1) gene. The specificity of these assays was validated with adult worms, naturally infected gastropods and human urine and stool samples. Sensitivity was tested on experimentally infected snail specimens that were sacrificed 10 and 40 days post-infection in order to mimic a natural prepatent and mature infection, respectively. The assay provides a diagnostic tool to support the xenomonitoring of planorbid gastropods for trematode infections in a One Health context, with a focus on the transmission monitoring of schistosomiasis.
\end{abstract}

Keywords: gastropod-borne disease, One Health, rapid diagnostic multiplex PCR, Schistosoma, transmission monitoring, Trematoda

\section{Introduction}

Schistosomiasis is the most prevalent gastropod-borne infectious disease in the world and causes an estimated 1.5 billion disability-adjusted life years (DALY) annually. ${ }^{1}$ The main pathology of Schistosoma-induced infections originates from migrating eggs that cause chronic lesions in a variety of organs. ${ }^{1}$ An estimated 192 million (93\%) of the worldwide estimated 207 million schistosomiasis cases occur in sub-Saharan Africa. ${ }^{2}$ Animal Schistosoma species are estimated to infect $>165$ million bovines in Africa and Asia, leading to substantial economic losses. ${ }^{3}$ Together with other gastropod-borne diseases like fascioliasis, echinostomiasis and amphistomiasis, animal schistosomiasis forms a major veterinary concern and an economic burden to the livestock industry by decreasing fertility, growth and calf survival. ${ }^{4,5}$ Schistosoma bovis, Schistosoma curassoni and Schistosoma mattheei are the most common Schistosoma species to infect livestock across sub-Saharan Africa. ${ }^{4}$ These species are also known to hybridize with Schistosoma haematobium, having important implications for schistosome epidemiology. ${ }^{6-8}$ Besides the burden for human health and the livestock industry, trematodes can be very abundant in terrestrial and aquatic ecosystems, sometimes exceeding the total biomass of top predators, ${ }^{9}$ thereby significantly affecting ecosystem health. Moreover, several domestic bovine schistosomes also infect wild herbivores, and hybrid strains are of zoonotic importance. ${ }^{4,7,8} \mathrm{~A}$ 'One Health' approach focussing on both human and animal schistosomiasis (or trematodiases in general) is therefore desired to efficiently map trematode distribution and effectively tackle trematodiasis outbreaks in both human, domesticated and wild animal populations. ${ }^{3}$

Gastropod-borne diseases are confined by the distribution of the gastropod intermediate hosts. Insights into trematode infection rates in gastropods are thus of major epidemiological importance in trematode transmission studies. The classic method for diagnosing and quantifying trematode infections in

(c) The Author(s) 2019. Published by Oxford University Press on behalf of Royal Society of Tropical Medicine and Hygiene. All rights reserved. For permissions, please e-mail: journals.permissions@oup.com. 
gastropods is by inducing the natural emergence of cercariae, termed 'shedding'. However, morphological identification of larval trematode stages in gastropods is highly error-prone and difficult to impossible for genus- or species-level identification, due to the lack of diagnostic morphological traits, phenotypic plasticity and the occurrence of cryptic species. ${ }^{10,11}$ Moreover, shedding significantly underestimates the prevalence of both single and multiple species co - infections. ${ }^{12}$ This can be the result of low infection load, immature (prepatent) infections or gastropod mortality during the shedding experiment. ${ }^{12,13}$ Hence a reliable assessment of trematode infection prevalence requires a molecular diagnostic technique. ${ }^{12}$

Molecular xenomonitoring (MX) is the screening of disease vectors or intermediate hosts for the genetic material of the pathogens they transmit. ${ }^{13}$ There is an ongoing debate whether polymerase chain reaction (PCR) or loop-mediated isothermal amplification (LAMP) is preferred for field-sample diagnostics, as each method is characterized by specific advantages and disadvantages. $^{14}$ LAMP has proven to be a suitable method for detecting early prepatent infections in gastropod hosts. ${ }^{15}$ With specificity 10 times higher than the classic PCR, LAMP requires minimal equipment and is regarded as a fast, sensitive and cheap tool for field diagnosis. ${ }^{15,16}$ However, false negative results are observed in LAMP assays as a consequence of the method's high sensitivity to inhibition by reagents used during deoxyribonucleic acid (DNA) extraction. ${ }^{15}$ Moreover, when compared with PCR, LAMP presents less versatility for multiplexing approaches. $^{17}$

Diagnostic multiplex PCR is a technique in which multiple genetic markers of differential length are amplified, allowing species-level identification up to species level. In this way, gastropod tissue samples can be screened for different trematode infections by separating the amplified DNA according to its length through agarose gel electrophoresis. ${ }^{18,19}$ Rapid diagnostic PCR (RD-PCR) refers to the use of PCR for direct diagnosis without the need for DNA sequencing, thus saving time and cutting costs.

Here we describe two PCR protocols that can be combined to identify infections by the most common African human- and livestock-infecting Schistosoma species in their planorbid gastropod host. The first multiplex PCR is an adaptation of the RD-PCR for screening Fasciola sp. infections in lymnaeid gastropods described by Carolus et al., ${ }^{20}$ in which we added primers for Schistosoma sp. detection in planorbids. This PCR screens for general trematode infections (all trematodes) and for infections with all species belonging to the Schistosoma genus, while amplifying an internal control to avoid false negative PCR results. When positive for Schistosoma sp. infection, the second multiplex PCR can be applied to discriminate S. haematobium, S. mansoni and S. mattheei from infections of S. bovis, S. curassoni and S. guineensis. This second RD-PCR is an optimization of the assays described by Webster et al. ${ }^{19}$ that differentiates $S$. haematobium from S. bovis and from Van den Broeck et al. ${ }^{21}$ that added a S. mansoni-specific primer to this assay. Here we added a newly designed reverse primer to differentiate $S$. mattheei, a bovine and occasionally human-infecting schistosome that is prevalent in southern Africa and is known to share common gastropod hosts with $S$. haematobium, S. bovis and $S$. intercalatum. ${ }^{4,6}$

\section{Materials and methods}

\section{Samples and DNA extraction}

To test and optimize the multiplex PCR assays we used experimentally infected gastropods (see 'Sensitivity testing'), naturally infected gastropods from the collection of the Royal Museum for Central Africa (RMCA, Tervuren, Belgium) and human urine and stool samples that tested positive for a $S$. haematobium and S. mansoni infection, respectively. The latter samples originate from a previous study in Senegal on schistosomiasis epidemiology, transmission and control (For more information see Van den Broeck et al. ${ }^{21}$ and Meurs et al. ${ }^{22}$ ). The naturally infected gastropods were collected in an ongoing study in South Africa and Zimbabwe (unpublished data). In both field studies, gastropods were tested for infection by means of shedding experiments and different cercarial types that were released after light stimulus were stored in ethanol and barcoded. For snail DNA extraction, whole soft tissue was separated from the shell, ethanol was removed and the tissue was subsequently homogenized with a sterile scalpel. The total DNA of homogenates was extracted using the E.Z.N.A. Mollusc DNA Kit (OMEGA Bio-tek, Norcross, GA, USA), specially designed for DNA extraction from tissue rich in mucopolysaccharides. All DNA extracts were stored at $-20^{\circ} \mathrm{C}$ after being diluted by a factor 10 in Milli-Q water (Merck, Darmstadt, Germany).

\section{Primer design}

The new COX1 and ITS2 primers were designed and tested in silico in Geneious 6.1.8 software (Geneious, Auckland, New Zealand) on alignments of gastropod and trematode sequences (representatives from all genera of African trematodes and for each species of Schistosoma available on GenBank Supplementary Table 1) downloaded from the National Center for Biotechnology Information (NCBI) GenBank (https://www. ncbi.nlm.nih.gov/). Primers included in the same multiplex PCR were analysed together in silico to predict the formation of potential primer dimers using the Thermo Fisher Scientific Multiple Primer Analyzer (online tool, https://www.thermofisher. com). The target region, length and sequence of each primer used are described in Table 1. Primer pairs were subsequently tested in simplex and multiplex PCR on gastropod specimens with known infection status in the two-step approach. In the first PCR, referred to as 'infection RD-PCR', three markers are amplified. Carolus et al. ${ }^{20}$ tested and described the use of the general trematode and gastropod internal control markers. This assay was adapted for Schistosoma detection via the addition of the newly designed Schistosoma sp. primer pair.

The second PCR, referred to as the 'Schistosoma RD-PCR', was designed for species discrimination of certain Schistosoma species and is only applied upon Schistosoma sp. detection in the infection RD-PCR. A universal forward primer, Asmit $1,{ }^{23}$ is combined with species-specific reverse primers (Sh.R for S. haematobium, Sman.R for S. mansoni, Smat.R for S. mattheei and Sb.R for S. bovis/S. curassoni/S. guineensis) that target markers of different lengths in the mitochondrial cytochrome c oxidase subunit 1 (COX1) gene. This is an optimization of the protocol described in Van den Broeck et al., ${ }^{21}$ originally based on Webster et al., ${ }^{19}$ 
Table 1. Primers used in the multiplex RD-PCR protocols. The primer sequences and names are given along with the targeted marker, target organism and length of the amplicon (length in the number of nucleotides). The symbol ' \pm ' indicates a possible variation in amplicon length because of interspecific variation.

\begin{tabular}{|c|c|c|c|c|c|}
\hline Primer name & Primer sequence $\left(5^{\prime}-3^{\prime}\right)$ & Marker & Length & Target organism & Published by \\
\hline \multicolumn{6}{|c|}{ Infection RD-PCR } \\
\hline 18S_SNAIL_F & AGTATGGTTGCAAAGCTGAAACTTA & $18 \mathrm{~S}$ & \pm 500 & Gastropoda spp. & Carolus et al. ${ }^{20}$ \\
\hline 18S_Digenea_F & CAGCTATGGTTCCTTAGATCRTA & \multirow[t]{2}{*}{$18 \mathrm{~S}$} & \multirow[t]{2}{*}{ \pm 392} & \multirow[t]{2}{*}{ Trematoda spp. } & \multirow[t]{2}{*}{ Carolus et al. ${ }^{20}$} \\
\hline 18S_Digenea_R & TATTTTTCGTCACTACCTCCCCGT & & & & \\
\hline ITS2_Schisto_F & GGAAACCAATGTATGGGATTATTG & \multirow[t]{2}{*}{ ITS2 } & \multirow[t]{2}{*}{ \pm 369} & \multirow[t]{2}{*}{ Schistosoma spp. } & \multirow[t]{2}{*}{ This study } \\
\hline Schistosoma $\mathrm{RD}$ & & & & & \\
\hline Asmit1 & TTTTTTGGTCATCCTGAGGTGTAT & \multirow[t]{5}{*}{$\operatorname{cox} 1$} & - & \multirow{5}{*}{$\begin{array}{l}\text { Schistosoma spp. } \\
\text { S. haematobium } \\
\text { S. mansoni } \\
\text { S. mattheei } \\
\text { S. bovis }\end{array}$} & \multirow{2}{*}{$\begin{array}{l}\text { Littlewood et al. }^{23} \\
\text { Webster et al. }^{19}\end{array}$} \\
\hline Sh.R & TGATAATCAATGACCCTGCAATAA & & 543 & & \\
\hline Sman.R & TGCAGATAAAGCCACCCCTGTG & & 375 & & Van den Broeck et al. ${ }^{21}$ \\
\hline Smat.R & CACCAGTTACACCACCAACAGA & & 362 & & This study \\
\hline Sb.R & CACAGGATCAGACAAACGAGTACC & & 306 & & Webster et al. ${ }^{19}$ \\
\hline
\end{tabular}

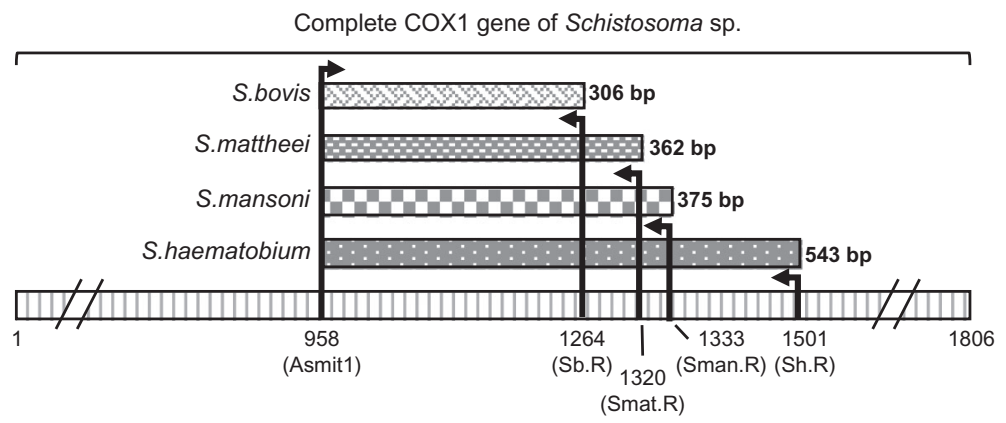

Figure 1. Schematic representation of the primers used in the Schistosoma RD-PCR and the primer location within the COX1 gene.

by adding a newly designed S. mattheei-specific primer. The species-specific reverse primers amplify bands of $S$. haematobium (543 bp), S. mansoni (375 bp), S. mattheei (362 bp) and S. bovis/ S. curassoni/S. guineensis (306 bp), respectively. A schematic representation of the target regions on COX1 and the respective positions of the different primers are presented in Figure 1.

\section{PCR amplification and visualisation}

All PCR reactions were performed in a $15 \mu \mathrm{L}$ volume with $2 \mu \mathrm{L}$ undiluted gastropod DNA extract using the Multiplex Taq DNA polymerase kit (Qiagen, Venlo, The Netherlands). Primer concentrations in the final volume were $0.2 \mu \mathrm{M}$ 18S_Digenea_F and 18S_Digenea_R, $0.1 \mu \mathrm{M}$ 18S_SNAIL_F and 18S_SNAIL_R and $0.6 \mu \mathrm{M}$ ITS2_Schisto_F and ITS2_Schisto_R in the infection RDPCR. The Schistosoma RD-PCR primer concentrations in the final volume were $0.8 \mu \mathrm{M}$ of Asmit1 universal forward primer $^{23}$ and $0.3 \mu \mathrm{M}$ of each reverse primer (Sh.R, Sb.R, Sman.R and the newly designed Smat.R primer, respectively). Both PCR protocols are performed with initial denaturation at $95^{\circ} \mathrm{C}$ for $3 \mathrm{~min}, 39$ cycles of $94^{\circ} \mathrm{C}$ for $30 \mathrm{~s}, 62^{\circ} \mathrm{C}$ for $45 \mathrm{~s}, 72^{\circ} \mathrm{C}$ for $45 \mathrm{~s}$ and a final elongation step at $72^{\circ} \mathrm{C}$ for $10 \mathrm{~min}$ in a TProfessional Thermal Cycler (Biometra, Göttingen, Germany). Analysis of the PCR product was carried out by gel electrophoresis for $1 \mathrm{~h} 45 \mathrm{~min}$ at 120 $\mathrm{V}$ in a $3 \%$ agarose gel with Midori Green direct staining and visualization using ultraviolet light.

\section{Validation and specificity testing}

The specificity of the infection RD-PCR primers was tested on a variety of infected gastropod species from the RMCA museum collection: Biomphalaria pfeifferi infected with S. mansoni, B. pfeifferi infected with Uvulifer sp., Bulinus globosus infected with Petasiger sp., Bulinus africanus infected with S. mattheei, B. africanus with a double infection of S. mattheei and Petasiger variospinosus, Lymnaea natalensis infected with $P$. variospinosus and $L$. natalensis infected with Echinostoma sp. Infections were confirmed by sequencing COX1, ITS and 18S rDNA of the trematode infections as described in Carolus et al. ${ }^{20}$ The specificity of the Schistosoma RD-PCR was tested using DNA of S. bovis, S. curassoni and S. guineensis (adult worm DNA provided by Dr Bonnie L. Webster), S. mansoni and S. haematobium (from human stool 
and urine samples, respectively), S. mattheei (cercariae from an infected B. africanus gastropod) and S. edwardiense (from an infected Biomphalaria sp.). The specificity of the primers Sh.R, Sb. $\mathrm{R}$ and Sman.R with S. intercalatum, S. margrebowiei, S. leiperi and S. rodhaini was already tested by Webster et al. ${ }^{19}$ and Van den Broeck et al. ${ }^{21}$. The new Smat.R primer was in silico tested for annealing with other schistosome COX1 sequences obtained from NCBI GenBank while using Geneious 6.1 .8 software (Supplementary Table 1).

The following parameters were assessed to validate both PCR assays: in silico specificity of all primers, trematode species specificity of infections in different gastropod species, the capability to detect Schistosoma spp. infections in case of co-infection and the probability of false positive and false negative results. Both PCR protocols were tested at annealing temperatures of $58^{\circ} \mathrm{C}, 62^{\circ} \mathrm{C}$ and $65^{\circ} \mathrm{C}$ to validate temperature-dependent specificity and optimal annealing conditions.

\section{Sensitivity testing}

The sensitivity of both PCR protocols was tested using pure DNA extracts of Biomphalaria glabrata experimentally exposed to S. mansoni and serial dilutions thereof. The experimentally infected gastropods comprised two B. glabrata from Brazil individually exposed to a single miracidium of S. mansoni from Brazil for 24 h. These gastropods were maintained at a temperature of around $25^{\circ} \mathrm{C}$, with a $12 \mathrm{~h} / 12 \mathrm{~h}$ photoperiod and fed ad libitum, after which they were sacrificed at 10 and $40 \mathrm{~d}$ postmonomiracidial exposure. We prefer this method over artificially mixed host and parasite DNA, as it better reflects natural infection dynamics. The gastropods were removed from their shell and preserved in 95\% ethanol. The concentration of DNA in the original DNA extract was measured using Qubit Fluorometric Quantitation (Thermo Fisher Scientific, Waltham, MA, USA). A dilution series of $10 \mathrm{ng}, 1 \mathrm{ng}, 100 \mathrm{pg}, 10 \mathrm{pg}, 1 \mathrm{pg}, 100 \mathrm{fg}, 10 \mathrm{fg}$ and $1 \mathrm{fg}$ DNA per microliter was made and PCR protocols were run.

\section{Results}

\section{Validation and specificity}

The infection RD-PCR was validated using different gastropods with and without Schistosoma sp. infections. Figure 2 shows the expected amplicons in an uninfected gastropod (Bulinus sp.) and gastropods infected with Schistosoma sp. (B. pfeifferi infected with S. mansoni) and non-Schistosoma trematodes ( $B$. globosus infected with Petasiger sp.). DNA extracts of $B$. africanus infected with S. mattheei, B. africanus with a double infection of S. mattheei and Petasiger variospinosus, L. natalensis infected with $P$. variospinosus, L. natalensis infected with Echinostoma sp. and $B$. pfeifferi infected with Uvulifer sp. all show the expected signals with the amplicon length corresponding to the internal control and general trematode markers (results not shown). The Schistosoma RD-PCR was shown to be a reliable diagnostic tool to identify infections of S. mansoni, S. haematobium and S. mattheei to the species level and to discriminate these species from S. bovis, S. curassoni and S. guineensis, as shown in Figure 3. The newly designed $S$. mattheei reverse primer (Smat.R) can specifically detect $S$. mattheei infections in their planorbid gastropod host, while it did not amplify in DNA samples from $S$. haematobium, S. edwardiense, S. mansoni, S. bovis, S. curassoni and S. guineensis. Due to a lack of DNA templates for the remaining Schistosoma species, we tested the specificity of the Smat.R primer in silico. These tests showed that the primer has at least $22.7 \%$ mismatching base pairs with all other schistosome species except for S. nasale (13.6\%; only occurring in Asia), S. edwardiense (13.6\%) and S. hippopotami (18.2\%). The fact that it did not amplify in our S. edwardiense DNA sample, which showed $13.6 \%$ mismatching base pairs, suggests that it will also most likely not

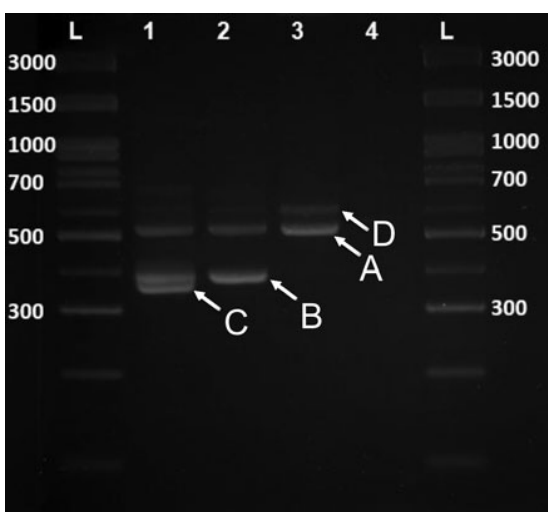

Figure 2. Results of the infection RD-PCR assay containing different gastropod species with a variety of infections. The arrows and corresponding letters indicate the three different markers: (A) the internal control ( 500 base pairs [bp]), (B) the general trematode marker ( 392 bp) and (C) the Schistosoma sp. marker ( 369 bp) as well as (D) aspecific amplicon. Lane 1 contains DNA of B. pfeifferi infected with S. mansoni, lane 2 contains B. globosus infected with Petasiger sp., lane 3 contains an uninfected Bulinus sp. and lane 4 contains a negative-PCR control (water). L: DNA ladder (300-3000 bp).

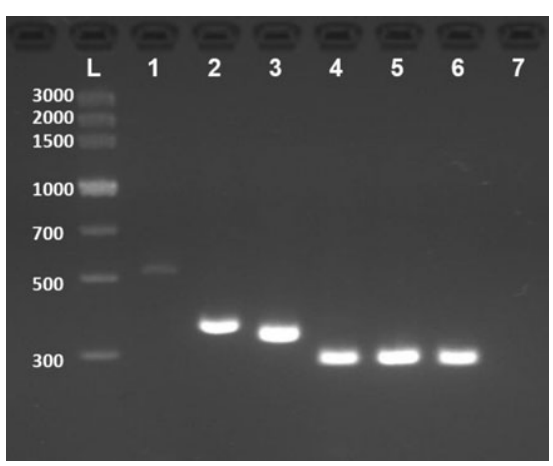

Figure 3. The Schistosoma RD-PCR results for S. haematobium (543 base pairs [bp]) in lane 1, S. mansoni (375 bp) in lane 2, S. mattheei (362 bp) in lane 3 , S. bovis (306 bp) in lane 4, S. curassoni (306 bp) in lane 5 and S. guineensis (306 bp) in lane 6 . Lane 7 contains a DNA extract from a field-caught, uninfected B. africanus. S. bovis, S. guineensis and S. curassoni DNA originates from isolated adult worm specimens, the $S$. haematobium extract originates from an infected human urine sample, the $S$. mansoni extract originates from an infected human stool sample and the S. mattheei DNA originates from an infected field-caught B. africanus. Species identity was confirmed by genotyping in all samples used. L: DNA ladder (300-3000 bp). 
amplify in the remaining schistosome species, which show more mismatching base pairs. The $S$. bovis reverse primer (Sb.R) also amplified in the closely related species $S$. guineensis and S. curassoni, as already pointed out by Webster et al. ${ }^{19}$ (see below). Both RD-PCR protocols show optimal amplification and specificity at $62^{\circ} \mathrm{C}$ (results not shown). We note the presence of aspecific amplification in some instances, as shown in Figure 2 (band D). We are confident that this is not the result of a co-infection, as it is also present in the experimentally infected gastropod (40-d infection) in Figure 4A. Also, its presence does not interfere with the interpretation of the infection status.

\section{Sensitivity}

The results for the sensitivity assays of the infection RD-PCR are shown in Figure 4A. The detection limit of the Schistosoma sp. marker in the experimentally infected $B$. glabrata was $1 \mathrm{ng} / \mu \mathrm{L}$ for an infection of $10 \mathrm{~d}$ and $10 \mathrm{pg} / \mu \mathrm{L}$ for a 40-day-old infection, respectively. The fact that the signal intensifies in the $40-\mathrm{d}$ post- exposure treatment compared with the 10-d post-exposure condition shows development and replication of S. mansoni within the snail during the course of infection. The general trematode marker showed a slightly higher sensitivity compared with the Schistosoma sp. marker and was still detectable in a total DNA dilution of $1 \mathrm{pg} / \mu \mathrm{L}$ and $100 \mathrm{fg} / \mu \mathrm{L}$ for an infection of $10 \mathrm{~d}$ and $40 \mathrm{~d}$, respectively. The sensitivity of the S. haematobium and S. bovis primer pairs of the Schistosoma RD-PCR was rigorously tested by Webster et al. ${ }^{19}$ In Figure 4B we show that the limit of detection in the Schistosoma RD-PCR is $100 \mathrm{pg} / \mu \mathrm{L}$ and $10 \mathrm{pg} / \mu \mathrm{L}$ for 10 - and 40-day-old S. mansoni infections, respectively.

\section{Discussion}

Molecular methods are indispensable for both trematode species discrimination ${ }^{10,11}$ and infection prevalence assessment. ${ }^{12}$ Because of their sensitivity and specificity, PCR-based techniques can be regarded as one of the most reliable, and perhaps the
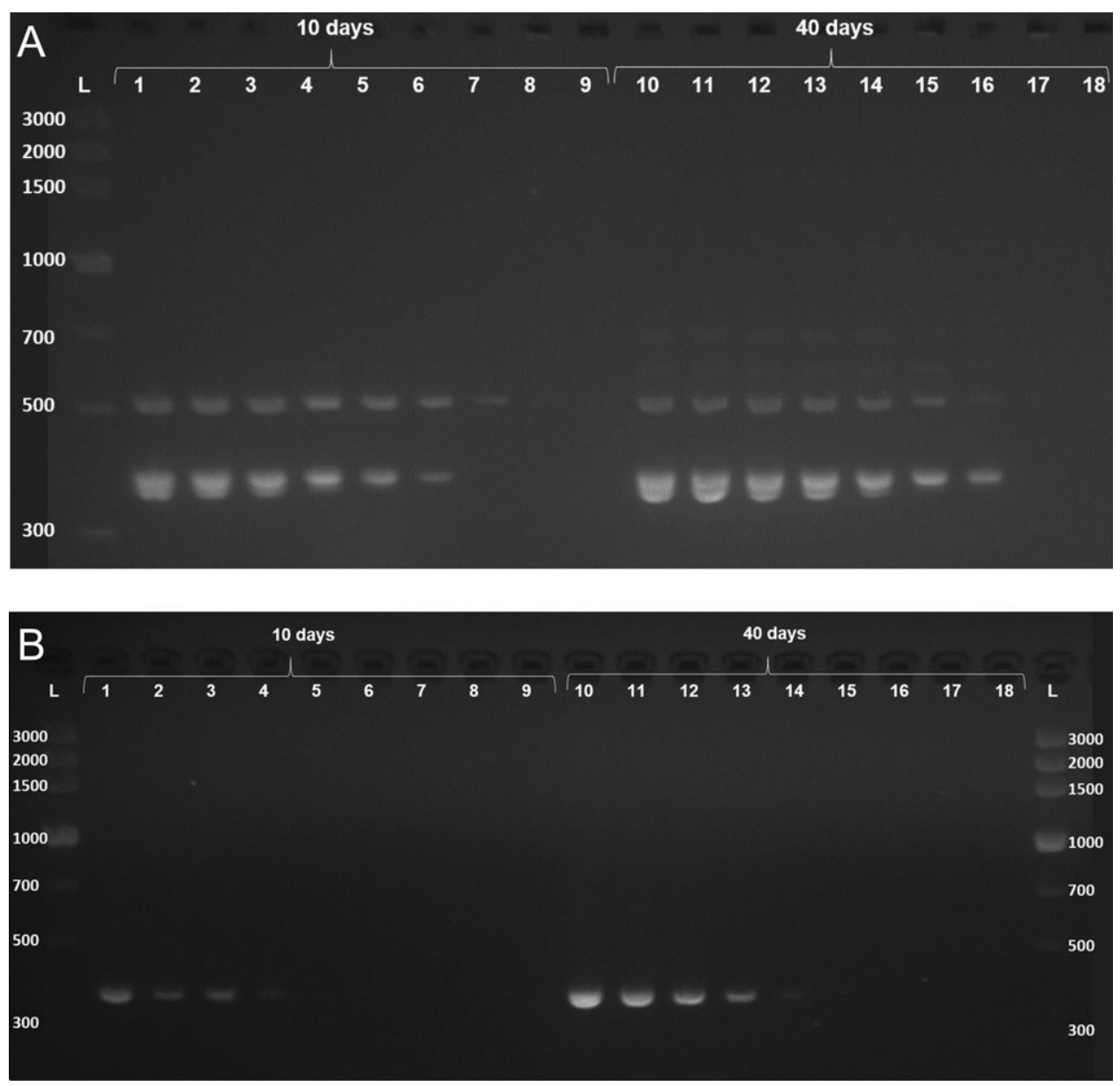

Figure 4. Sensitivity experiment for the (A) infection RD-PCR and (B) Schistosoma RD-PCR using experimentally infected B. glabrata with S. mansoni, sacrificed after $10 \mathrm{~d}$ (in lanes 1-9) or $40 \mathrm{~d}$ (in lanes 10-18) post-monomiracidial exposure. Both DNA extracts were used in undiluted form (total DNA concentration was $32.8 \mathrm{ng} / \mu \mathrm{L} 10 \mathrm{~d}$ post-infection and $76.7 \mathrm{ng} / \mu \mathrm{L} 40 \mathrm{~d}$ post-infection) and a dilution series of $10 \mathrm{ng}$ (lanes 2 and 11 ), $1 \mathrm{ng}$ (lanes 3 and 12), $100 \mathrm{pg}$ (lanes 4 and 13), $10 \mathrm{pg}$ (lanes 5 and 14), $1 \mathrm{pg}$ (lanes 6 and 15), $100 \mathrm{fg}$ (lanes 7 and 16), 10 fg (lanes 8 and 17) and $1 \mathrm{fg}$ (lanes 9 and 18) per microliter. L: DNA ladder (300-3000 bp). 
R. Schols et al.

golden standard, of diagnostic tools for detecting specific trematode species in their intermediate host, so-called xenomonitoring. ${ }^{12-14,16}$ Here we have developed a two-step approach to firstly detect trematode infections and then to discriminate the Schistosoma spp. involved in the gastropod infections. The 18S rDNA gene was chosen to design an internal control and a trematode-specific marker ${ }^{20}$ since it is a highly conserved region, ideal for the diagnosis of higher taxonomic levels such as the class Trematoda. ${ }^{10,24}$ The ITS2 region was targeted for Schistosoma sp. infection diagnosis because it is more variable and is capable of providing genus-specific primer annealing sites. $^{25}$ The infection RD-PCR was proven to be a valid diagnostic approach regarding its specificity: Schistosoma-specific bands in gastropods with a double trematode infection including Schistosoma sp. indicate that a second trematode infection in the gastropod sample does not interfere with the detection capabilities of the infection RD-PCR method. This proves its usefulness in the diagnostic screening of field-collected gastropods, in which co-infections commonly occur. ${ }^{12}$ The combination of the Schistosoma-specific and general trematode markers in the infection RD-PCR is also a highly valuable combination to simultaneously detect Schistosoma and other economically significant trematodes such as Paramphistomoidea (a major superfamily of trematodes that infect livestock) and Diplostomoidae (a superfamily of trematodes that infect birds and fish) that use the same planorbid hosts as schistosomes. ${ }^{4,26}$

The Schistosoma RD-PCR, on the other hand, is based on COX1, a marker that is extensively used for species-level identification of animals, as this haploid-inherited mitochondrial gene evolves rapidly compared with nuclear genes in the context of speciation. ${ }^{27}$ In the Schistosoma RD-PCR, at least three species of Schistosoma occurring in sympatry can readily be detected (i.e. S. mansoni, S. haematobium and S. mattheei). This is an optimization of the protocol described by Van den Broeck et al., ${ }^{21}$ based initially on Webster et al., ${ }^{19}$ in which S. mattheei, one of the most important livestock schistosomes in Africa, could not be discriminated. Our new Smat.R primer appears highly specific: it did not amplify in S. haematobium, S. edwardiense, S. mansoni, S. bovis, S. curassoni or S. guineensis. Ideally, specificity tests should be conducted on all Schistosoma spp. but the fact that it did not amplify in S. edwardiense, which showed $13.6 \%$ mismatching base pairs with Smat.R, suggests that it will most likely not amplify in the remaining schistosome species, which show more mismatching base pairs in the primer annealing region. We confirmed amplification of the previously published primer Sb.R in S. guineensis and S. curassoni. This is due to their close phylogenetic relationship with $S$. bovis and the associated lack of sufficient COX1 sequence variability for primer design. ${ }^{19}$ Increasing the annealing temperature to $65^{\circ} \mathrm{C}$ resolves this cross amplification but decreases the sensitivity of the protocol, as tested by Webster et al. ${ }^{19}$ S. curassoni and S. bovis infect livestock while $S$. guineensis infects humans in confined regions of West Africa. ${ }^{4}$ In the few geographic areas where $S$. bovis, S. curassoni and S. guineensis occur in sympatry, amplicon sequencing should confirm the identity of these species, as suggested by Webster et al. ${ }^{19}$ We can conclude that the optimized Schistosoma RD-PCR allows identification of the most common human schistosome species present in Africa (S. mansoni and S. haematobium) from those that infect livestock (S. bovis, S. curassoni and S. mattheei) in areas where S. guineensis is not present. It can be applied on stool or urine samples or on snail samples.

The serial dilution of DNA from lab-reared B. glabrata gastropods exposed to $S$. mansoni showed that the PCR assays can detect parasite DNA in both mature (40-day-old) and prepatent (10-dayold) gastropod infections at DNA concentrations below the values usually obtained after DNA extraction. The general trematode marker of the infection RD-PCR appeared to be the most sensitive marker, being 100 times more sensitive (for 40-day-old infections) than the Schistosoma-specific marker used in the same assay, and the Schistosoma RD-PCR. In all the assays, the detection limit increased 10- to 100-fold in 40-day-old infections compared with 10-day-old infections. Trematodes reproduce asexually within the gastropod host, so the relative abundance of trematode DNA increases with the age of the infection. The high sensitivity of this diagnostic multiplex RD-PCR approach suggests that this assay might also be a reliable diagnostic tool for the detection of trematode DNA in human excreta (see Figure 3), as also indicated by Van den Broeck et al., ${ }^{21}$ although standardized sensitivity and specificity tests are needed to validate this.

The infection RD-PCR described here is the first diagnostic PCR protocol for monitoring gastropod-borne trematode infections that includes a combination of an internal control, a general trematode marker and a Schistosoma-specific marker. The use of an internal control is an important asset in PCR-based diagnostics, because it excludes false negative results that commonly occur due to PCR inhibitors, failed DNA extractions, malfunctioning of thermal cyclers or an inconsistency in the preparation of the PCR mixture. ${ }^{28,29}$ Especially in studies where the sample size is large but the infection rate is low, false negative results can have a significant influence on the epidemiological conclusions drawn. ${ }^{29}$ We propose that an internal control should be a standard component of a diagnostic PCR protocol that is used in epidemiological studies of gastropod-borne diseases. The absence of an internal control in the Schistosoma RDPCR is justified through the two-step approach. Samples that, for example, would give a positive Schistosoma signal in the infection RD-PCR but fail to amplify a marker in the Schistosoma RD-PCR indicate an infection with a Schistosoma species other than the species accounted for in our Schistosoma RD-PCR, such as S. leiperi or S. margrebowiei. In that case, the identity of the infective Schistosoma species can be resolved by sequencing the amplicon of the infection RD-PCR.

The use of a general trematode marker is an interesting addition to standard PCR assays that only focus on one genus, such as Fasciola sp..$^{20,29}$ or Schistosoma sp. ${ }^{16,19}$ Other taxa that potentially form a threat to humans and domesticated or wild animals are not detected in these studies and thus remain unexplored. Including a general trematode marker, although invaluable in one study (e.g. the schistosomiasis monitoring program), can be extremely valuable for other study groups that focus on other particular trematodiases (e.g. fish trematodiases). This approach fits into the One Health framework for studying neglected tropical diseases. Since trematodiases affect the health of humans, livestock (such as cattle and farmed fish) and wild animals, they can be regarded as a burden for public, economic and environmental health. Also, the Schistosoma RDPCR fits the One Health approach as it identifies human and 
animal schistosomes. Both assays will therefore support the monitoring of trematode infections in different types of field samples.

\section{Supplementary data}

Supplementary data are available at Transactions online.

Authors' contributions: RS, HC and TH conceived the study. RS, HC, SM and $\mathrm{TH}$ designed the study protocol. RS, $\mathrm{HC}, \mathrm{CH}$ and $\mathrm{TH}$ carried out the RD-PCR assays and analysed and interpreted the data. RS and HC drafted the manuscript. $\mathrm{CH}, \mathrm{SM}, \mathrm{AM}$ and $\mathrm{TH}$ critically revised the manuscript for intellectual content. RS and $\mathrm{HC}$ contributed equally to this work. All authors read and approved the final manuscript.

Acknowledgements: We thank our colleagues from the University of Perpignan for the experimental infection of gastropod specimens, which were used in sensitivity screening. Furthermore, we would like to thank Tom Pennance and Bonnie L. Webster for their constructive comments, and thank Bonnie L. Webster and the Schistosomiasis Collection at the Natural History Museum (SCAN, London, UK) for providing the S. haematobium, S. bovis, S. curassoni and S. guineensis adult worms.

Funding: This work was funded by the Belgian Federal Science Policy Office (BRAIN-be Pioneer Project BR/165/PI/TRAIL) and the project S1_ZMB SNAILS SUPPORT 2017 of the annual programme of the Cooperation Agreement dd.18.06.07 between the Belgian Development Cooperation and the Royal Museum for Central Africa. CH is funded by an FWO fellowship grant of the Research Foundation-Flanders (FWO-Vlaanderen).

Competing interests: None declared.

Ethical approval: All experiments were performed according to the French national ethical standards established in 1 February 2013 (NOR: AGRG1238753A). Authorization was received from the French Ministere de l'Agriculture et de la Peche and the French Ministere de l'Education Nationale de la Recherche et de la technologie, permit A66040 (authorization 007083, decree 87-848).

\section{References}

1 Gryseels B, Polman K, Clerinx J, et al. Human schistosomiasis. Lancet. 2006;368(9541):1106-18.

2 Steinmann P, Keiser J, Bos R, et al. Schistosomiasis and water resources development: systematic review, meta-analysis, and estimates of people at risk. Lancet Infect Dis. 2006;6(7):411-25.

3 Gower CM, Vince L, Webster JP. Should we be treating animal schistosomiasis in Africa? The need for a one health economic evaluation of schistosomiasis control in people and their livestock. Trans R Soc Trop Med Hyg. 2017;111(6):244-7.

4 Toledo R, Fried B, editors. Digenetic trematodes. New York: Springer; 2014.

5 You H, Cai P, Tebeje B, et al. Schistosome vaccines for domestic animals. Trop Med Infect Dis. 2018;3(2):68.
6 Mahmoud AF, editor. Schistosomiasis. London: Imperial College Press; 2001.

7 Huyse T, Webster BL, Geldof S, et al. Bidirectional introgressive hybridization between a cattle and human schistosome species. PLoS Pathog. 2009;5(9):e1000571.

8 Leger E, Webster JP. Hybridizations within the genus Schistosoma: implications for evolution, epidemiology and control. Parasitology. 2017;144(1):65-80.

9 Kuris AM, Hechinger RF, Shaw JC, et al. Ecosystem energetic implications of parasite and free-living biomass in three estuaries. Nature. 2008;454(7203):515-8.

10 Nolan MJ, Cribb TH. The use and implications of ribosomal DNA sequencing for the discrimination of digenean species. Adv Parasitol. 2005;60:101-63.

11 Moszczynska A, Locke SA, McLaughlin JD, et al. Development of primers for the mitochondrial cytochrome $c$ oxidase I gene in digenetic trematodes (Platyhelminthes) illustrates the challenge of barcoding parasitic helminths. Mol Ecol Resour. 2009;9(Suppl s1):75-82.

12 Born-Torrijos A, Poulin R, Raga J, et al. Estimating trematode prevalence in snail hosts using a single-step duplex PCR: how badly does cercarial shedding underestimate infection rates? Parasit Vectors. 2014;7:243.

13 Caron Y, Rondelaud D, Losson B. The detection and quantification of a digenean infection in the snail host with special emphasis on Fasciola sp. Parasitol Res. 2008;103(4):735-44.

14 Pilotte N, Unnasch TR, Williams TA. The current status of molecular xenomonitoring for lymphatic filariasis and onchocerciasis. Trends Parasitol. 2017;33(10):788-98.

15 Fernández-Soto P, Gandasegui J, Sánchez HA, et al. A loop-mediated isothermal amplification (LAMP) assay for early detection of Schistosoma mansoni in stool samples: a diagnostic approach in a murine model. PLoS Negl Trop Dis. 2014;8(9):e3126.

16 Hamburger J, Abbasi I, Kariuki C, et al. Evaluation of loop-mediated isothermal amplification suitable for molecular monitoring of schistosome-infected snails in field laboratories. Am J Trop Med Hyg. 2013;88(2):344-51.

17 Gandasegui J, Fernández-Soto P, Hernández-Goenaga J, et al. Biompha-LAMP: a new rapid loop-mediated isothermal amplification assay for detecting Schistosoma mansoni in Biomphalaria glabrata snail host. PLoS Negl Trop Dis. 2016;10(12):e0005225.

18 Sahoo PR, Sethy K, Mohapatra S, et al. Loop mediated isothermal amplification: An innovative gene amplification technique for animal diseases. Vet World. 2016;9(5):465-9.

19 Webster BL, Rollinson D, Stothard JR, et al. Rapid diagnostic multiplex PCR (RD-PCR) to discriminate Schistosoma haematobium and S. bovis. J Helminthol. 2009;84(1):107-14.

20 Carolus H, Muzarabani KC, Hammoud C, et al. A cascade of biological invasions and parasite spillback in man-made Lake Kariba. Sci Total Environ. 2019;659:1283-92.

21 Van den Broeck F, Geldof S, Polman K, et al. Optimal sample storage and extraction protocols for reliable multilocus genotyping of the human parasite Schistosoma mansoni. Infect Genet Evol. 2011;11 (6):1413-8.

22 Meurs L, Mbow M, Boon N, et al. Micro-geographical heterogeneity in Schistosoma mansoni and S. haematobium infection and morbidity in a co-endemic community in northern Senegal. PLoS Negl Trop Dis. 2013;7(12):e2608.

23 Littlewood DTJ, Rohde K, Clough KA. Parasite speciation within or between host species? Phylogenetic evidence from site-specific polystome monogeneans. Int J Parasitol. 1997;27(11):1289-97. 
24 Routtu J, Grunberg D, Izhar R, et al. Selective and universal primers for trematode barcoding in freshwater snails. Parasitol Res. 2014; 113(7):2535-40.

25 Prokopowich CD, Gregory TR, Crease TJ. The correlation between rDNA copy number and genome size in eukaryotes. Genome. 2003;46(1):48-50.

26 Laidemitt MR, Zawadzki ET, Brant SV, et al. Loads of trematodes: discovering hidden diversity of paramphistomoids in Kenyan ruminants. Parasitology. 2017;144(2):131-47.
27 Hebert PDN, Cywinska A, Ball SL, et al. Biological identifications through DNA Barcodes. Proc Biol Sci. 2003;270(1512):313-21.

28 Hoorfar J, Cook N, Malorny B, et al. Diagnostic PCR: making internal amplification control mandatory. Lett Appl Microbiol. 2004;38(2): 79-80.

29 Caron Y, Righi S, Lempereur L, et al. An optimized DNA extraction and multiplex PCR for the detection of Fasciola sp. in lymnaeid snails. Vet Parasitol. 2011;178(1-2):93-9. 